\title{
Design and Analysis of a 2-D Eigenspace-Based Interference Canceller
}

\author{
Cheng-Chou Lee and Ju-Hong Lee, Member, IEEE
}

\begin{abstract}
This paper deals with the problem of eigenspacebased interference cancellation using a two-dimensional (2-D) rectangular array. An efficient 2-D signal blocking technique is presented to remove the desired signal from the received array data. In conjunction with the 2-D signal blocking technique, a positive definite matrix is further constructed and used to compensate the effect of the signal blocking operation on the sensor noise received by a 2-D eigenspace-based interference canceller (EIC). Therefore, the interference subspace required for computing the optimal weight vector of the designed 2-D EIC can be obtained by simply using conventional eigenvalue decomposition methods instead of any complicated generalized eigenvalue decomposition methods. The performances of the designed 2-D EIC under finite samples and steering angle error are also evaluated. The developed theoretical results are confirmed by several simulation examples.
\end{abstract}

Index Terms-Electromagnetic radiative interference, interference concellation.

\section{INTRODUCTION}

A DAPTIVE interference cancellation can be used for maximizing the rejection of interference regardless of the interference-to-noise ratio (INR) when processing array data. This goal can be efficiently achieved by utilizing eigenspacebased interference cancellers (EIC's) as presented in the literature [1]-[6]. A common feature for these EIC's is that the interference subspace (IS) spanned by the interferers must be first computed. Then, the optimal weight vector is computed by maximizing the output signal-to-noise power ratio (SNR) subject to a constraint of orthogonality to the IS.

Notable among these EIC's is the one presented by [4] due to its several advantages over the others. Using a onedimensional (1-D) uniformly linear array (ULA) and an appropriately designed signal blocking processor which blocks the desired signal from the received array data, it finds the IS through the generalized eigenvalue decomposition (GEVD) of the correlation matrix of the data vector at the output of the signal blocking processor. However, the noise component left in the blocked data vector is no longer spatially white because of using the signal blocking matrix. Hence, finding the required IS for computing the optimal weight vector inevitably resorts to a complicated GEVD. As a result, it is very difficult to evaluate the statistical performance under finite samples and

Manuscript received May 20, 1997; revised October 29, 1998. This work was supported by the National Science Council under Grant NSC85-2213E002-008.

The authors are with the Department of Electrical Engineering, National Taiwan University, Taipei 106, Taiwan.

Publisher Item Identifier S 0018-926X(99)04782-1. the robust capability against steering angle error for the EIC. Moreover, the technique presented in [4] cannot be extended to process two-dimensional (2-D) array data since its 1-D blocking scheme can not be directly applied to the 2-D case. In the literature, there are practically no papers dealing with eigenspace-based interference cancellation using 2-D adaptive arrays.

In this paper, we present the theoretical results for designing and analyzing an EIC using a 2-D adaptive array. Two 1-D blocking matrices are first designed for both row and column subarrays, respectively. Using the blocked data vectors at the output of these 1-D blocking matrices and the properties of Kronecker product for matrices, a 2-D blocking technique is developed to construct a blocked data correlation matrix $R$ that does not contain the desired signal component for computing the IS. However, the noise component in $R$ is no longer spatially white, which introduces more complexity in computing the IS. To eliminate this effect, a positive definite matrix $\Omega$ is created from the designed blocking scheme and $\pi_{n} \Omega$ is then added to $R$, where $\pi_{n}$ is the background noise power. The resulting data correlation matrix $R+\pi_{n} \Omega$ then possesses a noise component which is spatially white. As a result, we can find an orthogonal basis matrix of the IS by performing the conventional EVD and then construct the optimal weight vector using this orthogonal basis matrix. This technique facilitates the analyses of the statistical performance under finite samples and the robust capability against steering angle error for the 2-D EIC. Theoretical results on the expectation of the output signal-to-interference plus noise ratio (SINR) are presented for showing the statistical performance of the 2-D EIC. As to the robust capability against steering angle error, it is shown that the performance of the 2-D EIC may be significantly degraded even if there is a small steering angle error. However, using the proposed 2-D blocking technique with higher order can alleviate the difficulty. The breakdown threshold for the 2-D EIC's performance due to steering angle error is also derived.

This paper is organized as follows. Section II presents the design of an eigenspace-based interference canceller using a 2-D rectangular array. A 2-D blocking technique is developed and the construction of a positive definite matrix for eliminating the effect of the 2-D blocking operation on the spatially white noise received by the 2-D array is proposed. Section III analyzes the statistical performance under finite snapshots for the designed 2-D EIC. The performance of the designed 2-D EIC in the presence of steering angle error is evaluated in Section IV. Several simulation examples for illustration 
and confirmation of the theoretical works are provided in Section V. Finally, Section VI gives a conclusion for this paper.

\section{DESIGN OF A 2-D EIGENSPACE-BASED INTERFERENCE CANCELLER}

\section{A. The 2-D Array Data Model}

Consider a 2-D $\bar{M} \times \bar{N}$ uniform rectangular array (URA) with sensors located on the $X-Y$ plane at the positions $((m-1) \lambda / 2,(n-1) \lambda / 2)$ for $m=1,2, \ldots, \bar{M}$ and $n=$ $1,2, \ldots, \bar{N}$, where $\lambda$ represents the signal wavelength. Let the signal impinging on the array from the elevation angle $\theta$ and azimuth angle $\phi$ yield a unit magnitude response and a phase response given by $\exp \{j[(m-1) \pi u+(n-1) \pi v]\}$ at the array sensor located at $((m-1) \lambda / 2,(n-1) \lambda / 2)$, where $j=\sqrt{-1}$ and $(u, v)=(\sin (\theta) \cos (\phi), \sin (\theta) \sin (\phi)) . P$ narrow-band signals are impinging on the URA from $P$ distinct angles $\left(u_{i}, v_{i}\right)$ for $i=1,2, \ldots, P$. Thus, the data received by the sensor located at $((m-1) \lambda / 2,(n-1) \lambda / 2)$ can be expressed as

$$
\begin{aligned}
\bar{x}_{m, n}(t)= & \sum_{i=1}^{P} \bar{s}_{i}(t) \exp \left\{j \left[(m-1) \pi u_{i}\right.\right. \\
& \left.\left.+(n-1) \pi v_{i}\right]\right\}+\bar{y}_{m, n}(t)
\end{aligned}
$$

where $\bar{s}_{i}(t)$ denotes the complex waveform of the signal emitted by the $i$ th source and $\bar{y}_{m, n}(t)$ the spatially white sensor noise independent of $\bar{s}_{i}(t)$. Without loss of generality, assume that $\bar{s}_{1}(t)$ is the desired signal with direction angle $\left(u_{1}, v_{1}\right)$ and the other $P-1$ signals are interferers. From (1), the data matrix received by the URA is given by

$$
\bar{X}(t)=\sum_{i=1}^{P}\left[\bar{A}_{c}\left(u_{i}\right) \bar{A}_{r}\left(v_{i}\right)^{T}\right] \bar{s}_{i}(t)+\bar{Y}(t)
$$

where $\bar{A}_{c}\left(u_{i}\right)=\left[1, \exp \left\{j \pi u_{i}\right\}, \ldots, \exp \left\{j(\bar{M}-1) \pi u_{i}\right\}\right]^{T}$, $\bar{A}_{r}\left(v_{i}\right)=\left[1, \exp \left\{j \pi v_{i}\right\}, \ldots, \exp \left\{j(\bar{N}-1) \pi v_{i}\right\}\right]^{T}$, and $\bar{Y}(t)$ is the received noise matrix. Rewriting (2) in vector form, we have

$$
\begin{aligned}
\operatorname{vec}\{\bar{X}(t)\}= & {\left[\bar{x}_{1,1}(t), \ldots, \bar{x}_{\bar{M}, 1}(t), \bar{x}_{1,2}(t), \ldots, \bar{x}_{\bar{M}, 2}(t)\right.} \\
& \left.\bar{x}_{1,3}(t), \ldots, \bar{x}_{1, \bar{N}}(t), \ldots, \bar{x}_{\bar{M}, \bar{N}}(t)\right]^{T}
\end{aligned}
$$

Using the following property of Kronecker product [7]:

$$
\langle\mathrm{KP} .1\rangle \operatorname{vec}\left\{Q_{1} Q_{2} Q_{3}^{T}\right\}=\left(Q_{3} \otimes Q_{1}\right) \operatorname{vec}\left\{Q_{2}\right\}
$$

where $Q_{i}$ are matrices with appropriate sizes, we can rewrite (3) as

$$
\begin{aligned}
\operatorname{vec}\{\bar{X}(t)\} & =\sum_{i=1}^{P} \bar{A}\left(u_{i}, v_{i}\right) \bar{s}_{i}(t)+\operatorname{vec}\{\bar{Y}(t)\} \\
& =\bar{A}_{S} \bar{S}_{S}(t)+\operatorname{vec}\{\bar{Y}(t)\}
\end{aligned}
$$

where the response vector of the $i$ th signal source $\bar{A}\left(u_{i}, v_{i}\right)=$ $\bar{A}_{r}\left(v_{i}\right) \otimes \bar{A}_{c}\left(u_{i}\right)$, the response matrix of the $P$ signal sources
$\bar{A}_{S}=\left[\bar{A}\left(u_{1}, v_{1}\right) \cdots \bar{A}\left(u_{P}, v_{P}\right)\right]$, and the signal source vector $\bar{S}_{S}(t)=\left[\bar{s}_{1}(t), \ldots, \bar{s}_{P}(t)\right]^{T}$. The correlation matrix of $\operatorname{vec}\{\bar{X}(t)\}$ is then given by

$$
\bar{R}=E\left\{\operatorname{vec}\{\bar{X}(t)\} \operatorname{vec}\{\bar{X}(t)\}^{H}\right\}=\bar{A}_{S} \bar{\Psi}_{S} \bar{A}_{S}^{H}+\pi_{n} I_{\bar{M} \bar{N}}
$$

where $\bar{\Psi}_{S}=E\left\{\bar{S}_{S}(t) \bar{S}_{S}(t)^{H}\right\}$ denotes the full rank correlation matrix of the signal sources, $\pi_{n}$ the noise power, and $I_{\bar{M} \bar{N}}$ the $\bar{M} \bar{N} \times \bar{M} \bar{N}$ identity matrix.

\section{B. The 2-D Blocking Technique}

In the follwoing, we present a technique for the design of a 2-D EIC with a steering angle $\left(u_{0}, v_{0}\right)$. Utilizing the results presented in [4] and letting the steering angle be accordant with the direction angle $\left(u_{1}, v_{1}\right)$ of the desired signal, we can construct a blocking matrix $B_{c}$ for the column subarrays of the 2-D URA such that

$$
B_{c}^{H} \bar{A}_{c}\left(u_{i}\right)=d_{c, i} A_{c}\left(u_{i}\right) \quad \text { with } d_{c, i}=\left(e^{j \pi u_{i}}-e^{j \pi u_{1}}\right)^{\beta}
$$

and

$$
A_{c}\left(u_{i}\right)=J_{c} \bar{A}_{c}\left(u_{i}\right)
$$

where $\beta$ is the order of $B_{c} . J_{c}=\left[\begin{array}{ll}I_{M} & O_{M, \beta}\end{array}\right]$ is the row selection matrix which selects the first $M=\bar{M}-\beta$ rows of $\bar{A}_{c}\left(u_{i}\right)$, where $O_{m, n}$ is an $m \times n$ zero matrix. Similar results can be obtained for the row subarrays as

$$
B_{r}^{H} \bar{A}_{r}\left(v_{i}\right)=d_{r, i} A_{r}\left(v_{i}\right) \quad \text { with } d_{r, i}=\left(e^{j \pi v_{i}}-e^{j \pi v_{1}}\right)^{\delta}
$$

and

$$
A_{r}\left(v_{i}\right)=J_{r} \bar{A}_{r}\left(v_{i}\right)
$$

where $\delta$ is the order of $B_{r} . J_{r}=\left[\begin{array}{ll}I_{N} & O_{N, \delta}\end{array}\right]$ is the row selection matrix which selects the first $N=\bar{N}-\delta$ rows of $\bar{A}_{r}\left(v_{i}\right)$. Based on the above results, we present a 2-D blocking technique as follows.

Theorem 1: Let the matrix $R$ be given by

$$
R=\tilde{B}_{c}^{H} \bar{R} \tilde{B}_{c}+\tilde{B}_{r}^{H} \bar{R} \tilde{B}_{r}, \quad \text { with } \tilde{B}_{c}=J_{r}^{T} \otimes B_{c}
$$

and

$$
\tilde{B}_{r}=B_{r} \otimes J_{c}^{T}
$$

Then $R$ is an autocorrelation matrix of the blocked 2-D array data which contain all the interferers except the desired signal.

Proof: Based on the fact that $\tilde{B}_{c}=J_{r}^{T} \otimes B_{c}, \bar{A}\left(u_{i}, v_{i}\right)=$ $\bar{A}_{r}\left(v_{i}\right) \otimes \bar{A}_{c}\left(u_{i}\right)$, and the property of Kronecker product [7]

$$
\langle\mathrm{KP} .2\rangle \quad\left(Q_{1} \otimes Q_{2}\right)\left(Q_{3} \otimes Q_{4}\right)=\left(Q_{1} Q_{3}\right) \otimes\left(Q_{2} Q_{4}\right)
$$

it can be shown that

$$
\tilde{B}_{c}^{H} \bar{A}\left(u_{i}, v_{i}\right)=d_{c, i} A\left(u_{i}, v_{i}\right) .
$$

where $d_{c, i}$ is given by (6) and $A\left(u_{i}, v_{i}\right)=A_{r}\left(v_{i}\right) \otimes A_{c}\left(u_{i}\right)$. Based on (5) and (9), then we have

$$
\tilde{B}_{c}^{H} \bar{R} \tilde{B}_{c}=A_{S} \tilde{D}_{c} \bar{\Psi}_{S} \tilde{D}_{c}^{H} A_{S}^{H}+\pi_{n} \tilde{B}_{c}^{H} \tilde{B}_{c}
$$

where $A_{S}=\left[A\left(u_{1}, v_{1}\right), \ldots, A\left(u_{P}, v_{P}\right)\right]$ and $\tilde{D}_{c}=\operatorname{diag}$ $\left\{d_{c, 1}, \ldots, d_{c, P}\right\}$. Based on the fact of $d_{c, 1}=0$ and the property of KP.2, (10) can be further written as

$$
\tilde{B}_{c}^{H} \bar{R} \tilde{B}_{c}=A_{I} D_{c} \bar{\Psi}_{I} D_{c}^{H} A_{I}^{H}+\pi_{n}\left(I_{N} \otimes\left(B_{c}^{H} B_{c}\right)\right)
$$


where $A_{I}=\left[A\left(u_{2}, v_{2}\right), \ldots, A\left(u_{P}, v_{P}\right)\right], \quad D_{c}=\operatorname{diag}$ $\left\{d_{c, 2}, \ldots, d_{c, P}\right\}, \bar{\Psi}_{I}=E\left\{\bar{S}_{I}(t) \bar{S}_{I}(t)^{H}\right\}$, and $\bar{S}_{I}(t)=$ $\left[\bar{s}_{2}(t), \ldots, \bar{s}_{P}(t)\right]^{T}$. Similar to (11), we have the following result for the row subarrays:

$$
\tilde{B}_{r}^{H} \bar{R} \tilde{B}_{r}=A_{I} D_{r} \bar{\Psi}_{I} D_{r}^{H} A_{I}^{H}+\pi_{n}\left(\left(B_{r}^{H} B_{r}\right) \otimes I_{M}\right)
$$

where $D_{r}=\operatorname{diag}\left\{d_{r, 2}, \ldots, d_{r, P}\right\}$. Summing (11) and (12) yields

$$
R=\tilde{B}_{c}^{H} \bar{R} \tilde{B}_{c}+\tilde{B}_{r}^{H} \bar{R} \tilde{B}_{r}=A_{I} \Psi_{I} A_{I}^{H}+\pi_{n} \Phi
$$

where

$$
\Psi_{I}=D_{c} \bar{\Psi}_{I} D_{c}^{H}+D_{r} \bar{\Psi}_{I} D_{r}^{H}
$$

and

$$
\Phi=I_{N} \otimes\left(B_{c}^{H} B_{c}\right)+\left(B_{r}^{H} B_{r}\right) \otimes I_{M} .
$$

Clearly, $\Psi_{I}$ is a positive definite matrix if $\left(u_{i}, v_{i}\right) \neq\left(u_{1}, v_{1}\right)$ for all $i=2, \ldots, P$. From (13) to (15), we note that $R$ is the autocorrelation matrix of a data vector which does not contain the desired signal component.

\section{The 2-D EIC Formulation}

Based on the 1-D results of [4], the criterion in finding the optimal weight vector for the 2-D EIC can be defined as maximizing the output SNR subject to a constraint of orthogonality to the IS. Accordingly, we have to solve the following optimization problem:

$$
\text { Maximize } \frac{\left|W^{H} A\left(u_{1}, v_{1}\right)\right|^{2}}{W^{H} W} \quad \text { subject to } W \perp \operatorname{range}\left\{A_{I}\right\}
$$

where $A\left(u_{1}, v_{1}\right)$ serves as the steering vector. The optimal solution of (16) is given by

$$
W_{o}=\left(I_{M N}-A_{I}\left(A_{I}^{H} A_{I}\right)^{-1} A_{I}^{H}\right) A\left(u_{1}, v_{1}\right) .
$$

Equation (17) reveals that the matrix $A_{I}=\left[A\left(u_{2}, v_{2}\right), \ldots\right.$, $\left.A\left(u_{P}, v_{P}\right)\right]$ due to the $P-1$ interferers must be found in order to compute $W_{o}$. However, $A_{I}$ cannot be known a priori. Basically, one can resort to finding a basis matrix spanning range $\left\{A_{I}\right\}$ to solve this problem. Unfortunately, the matrix $\Phi$ given by (15) is generally not an identity matrix. Hence, we have to perform the GEVD of $R$. Let the generalized eigenvalues and the corresponding eigenvectors be designated as $\gamma_{i}$ and $g_{i}$, respectively. Accordingly, we have the following expression:

$$
R g_{i}=\gamma_{i} \Phi g_{i}
$$

where $\gamma_{1} \geq \gamma_{2} \geq \cdots \geq \gamma_{P-1}>\gamma_{P}=\cdots=\gamma_{M N}=\pi_{n}$. Let $G_{I}=\left[g_{1}, g_{2}, \ldots, g_{P-1}\right]$, then it can be shown that range $\left\{A_{I}\right\}=\operatorname{range}\left\{\Phi G_{I}\right\}$. Therefore, the optimal weight vector of (17) can be rewritten as

$W_{o}=\left(I_{M N}-\left(\Phi G_{I}\right)\left(\left(\Phi G_{I}\right)^{H}\left(\Phi G_{I}\right)\right)^{-1}\left(\Phi G_{I}\right)^{H}\right) A\left(u_{1}, v_{1}\right)$.

From (19), we note that performing the complicated GEVD of $R$ is inevitable for computing the optimal weight vector $W_{o}$.
Moreover, evaluating the statistical performance under finite data samples and the sensitivity to steering angle error for the 2-D EIC becomes very difficult because the GEVD of $R$ is necessary for designing the 2-D EIC.

To tackle the above two problems, in Appendix A, an efficient method is presented to construct such a positive definite matrix $\Omega$ that the effect of the 2-D blocking operation on the noise component of the received array data can be eliminated, i.e., $\Phi+\Omega=\sigma^{2} I_{M N}$. Thus, we obtain

$$
R_{w}=R+\pi_{n} \Omega=A_{I} \Psi_{I} A_{I}^{H}+\sigma^{2} \pi_{n} I_{M N} .
$$

Equation (20) reveals that the corresponding noise component in $R_{w}$ becomes spatially white. Performing the EVD of $R_{w}$, we have the following expression:

$$
R_{w} e_{i}=\lambda_{i} e_{i}
$$

where $\lambda_{1} \geq \lambda_{2} \geq \cdots \geq \lambda_{P-1}>\lambda_{P}=\cdots=\lambda_{M N}=\sigma^{2} \pi_{n}$. Let the matrix $E_{I}=\left[\begin{array}{lll}e_{1} & \cdots & e_{P-1}\end{array}\right]$ and the matrix $E_{R}=$ $\left[\begin{array}{lll}e_{P} & \cdots & e_{M N}\end{array}\right]$. It is easy to show that $\left[\begin{array}{ll}E_{I} & E_{R}\end{array}\right]^{H}\left[\begin{array}{ll}E_{I} & E_{R}\end{array}\right]=$ $I_{M N}$ and

$$
\operatorname{range}\left\{E_{I}\right\}=\operatorname{range}\left\{A_{I}\right\} \perp \operatorname{range}\left\{E_{R}\right\}
$$

i.e., $E_{I}$ is an orthogonal basis matrix spanning range $\left\{A_{I}\right\}$ and $E_{R}$ is an orthogonal basis matrix spanning the complement of range $\left\{A_{I}\right\}$. It follows from (22) that the optimal weight vector for the 2-D EIC based on the criterion of (16) can be rewritten as

$$
W_{o}=\left(I_{M N}-E_{I} E_{I}^{H}\right) A\left(u_{1}, v_{1}\right)=E_{R} E_{R}^{H} A\left(u_{1}, v_{1}\right) .
$$

\section{Statistical Performance UNDER FinIte Data SAMPLES}

In practice, the number of signal sources $P$, the background noise power $\pi_{n}$, and the ensemble correlation matrix $\bar{R}$ required for implementing the 2-D EIC are not available and usually estimated from the received data snapshots. Using the first $K$ data snapshots, we obtain the estimate $P$ for the number of signal sources based on the AIC or MDL criterion presented by [11]. Moreover, implementing the AIC or MDL criterion requires performing the EVD of the corresponding data correlation matrix. Therefore, $\pi_{n}$ can be estimated by utilizing the eigenvalue method of [12] during the same estimation process. Let the estimated value be denoted as $\hat{\pi}_{n}$. Then, the next $L$ data snapshots are used to compute the sample correlation matrix $\bar{R}$ as follows:

$$
\hat{\bar{R}}=\frac{1}{L} \sum_{l=1}^{L} \operatorname{vec}\left\{\bar{X}\left(t_{l}\right)\right\} \operatorname{vec}\left\{\bar{X}\left(t_{l}\right)\right\}^{H}
$$

to replace $\bar{R}$, where $\bar{X}\left(t_{l}\right)$ is the data matrix received at the time instant $t_{l}$. The correlation matrix $R_{w}$ of (20) is then replaced by

$$
\hat{R}_{w}=\hat{R}+\hat{\pi}_{n} \Omega
$$

where

$$
\hat{R}=\tilde{B}_{c}^{H} \hat{\bar{R}} \tilde{B}_{c}+\tilde{B}_{r}^{H} \hat{\bar{R}} \tilde{B}_{r}
$$


It is appropriate to assume that $\hat{\pi}_{n}$ and $\hat{R}$ are independent in this case. Thus, (21) becomes

$$
\hat{R}_{w} \hat{e}_{i}=\hat{\lambda}_{i} \hat{e}_{i}
$$

where $\hat{\lambda}_{1} \geq \hat{\lambda}_{2} \geq \cdots \geq \hat{\lambda}_{M N}$. Since the number of interferers is $P-1$, the corresponding basis matrix of IS and its complement are given by $\hat{E}_{I}=\left[\begin{array}{lll}\hat{e}_{1} & \cdots & \hat{e}_{P-1}\end{array}\right]$ and $\hat{E}_{R}=\left[\begin{array}{lll}\hat{e}_{P} & \cdots & \hat{e}_{M N}\end{array}\right]$, respectively. Consequently, the optimal weight vector for the 2-D EIC under finite snapshots is given by

$$
\hat{W}_{o}=\left(I_{M N}-\hat{E}_{I} \hat{E}_{I}^{H}\right) A\left(u_{1}, v_{1}\right)=\hat{E}_{R} \hat{E}_{R}^{H} A\left(u_{1}, v_{1}\right) \text {. }
$$

The statistical performance of the proposed 2-D EIC under finite data samples is given by the following theorem.

Theorem 2: For the case of input INR high enough, the expectation of the output SINR can be approximately given by

$$
E\left\{\widehat{\operatorname{SINR}}_{\circ}\right\} \approx \operatorname{SINR}_{\circ}\left(1-\frac{1}{L} F S P\right)
$$

where $\operatorname{SINR}_{o}$ denotes the array output SINR without the finite sample effect. FSP represents the factor of statistical performance and is given by

$$
F S P=\omega^{-1}\left(\xi_{r, r} \omega_{r, r}+\xi_{c, c} \omega_{c, c}+\xi_{r, c} \omega_{c, r}+\xi_{c, r} \omega_{r, c}\right)
$$

where $\omega=W_{o}^{H} W_{o}$

$$
\begin{cases}\omega_{c, c}=W_{o}^{H} \tilde{B}_{c}^{H} \tilde{B}_{c} W_{o}, & \omega_{r, r}=W_{o}^{H} \tilde{B}_{r}^{H} \tilde{B}_{r} W_{o} \\ \omega_{c, r}=W_{o}^{H} \tilde{B}_{c}^{H} \tilde{B}_{r} W_{o}, & \omega_{r, c}=W_{o}^{H} \tilde{B}_{r}^{H} \tilde{B}_{c} W_{o}\end{cases}
$$

and

$$
\left\{\begin{array}{l}
\xi_{c, c}=\operatorname{Tr}\left\{\Psi_{I}^{-1} \bar{\Psi}_{I} \Psi_{I}^{-1} D_{c} \bar{\Psi}_{I} D_{c}^{H}\right\} \\
\xi_{r, r}=\operatorname{Tr}\left\{\Psi_{I}^{-1} \bar{\Psi}_{I} \Psi_{I}^{-1} D_{r} \bar{\Psi}_{I} D_{r}^{H}\right\} \\
\xi_{c, r}=\operatorname{Tr}\left\{\Psi_{I}^{-1} \bar{\Psi}_{I} \Psi_{I}^{-1} D_{c} \bar{\Psi}_{I} D_{r}^{H}\right\} \\
\xi_{r, c}=\operatorname{Tr}\left\{\Psi_{I}^{-1} \bar{\Psi}_{I} \Psi_{I}^{-1} D_{r} \bar{\Psi}_{I} D_{c}^{H}\right\}
\end{array}\right\} .
$$

Proof: Please see Appendix B.

If the interferers are uncorrelated, (32) can be further simplified as

$$
\left\{\begin{array}{l}
\xi_{c, c}=\sum_{i=2}^{P}\left|d_{c, i}\right|^{2}\left(\left|d_{c, i}\right|^{2}+\left|d_{r, i}\right|^{2}\right)^{-2} \\
\xi_{r, r}=\sum_{i=2}^{P}\left|d_{r, i}\right|^{2}\left(\left|d_{c, i}\right|^{2}+\left|d_{r, i}\right|^{2}\right)^{-2} \\
\xi_{c, r}=\sum_{i=2}^{P} d_{c, i} d_{r, i}^{*}\left(\left|d_{c, i}\right|^{2}+\left|d_{r, i}\right|^{2}\right)^{-2} \\
\xi_{r, c}=\sum_{i=2}^{P} d_{r, i} d_{c, i}^{*}\left(\left|d_{r, i}\right|^{2}+\left|d_{c, i}\right|^{2}\right)^{-2}
\end{array}\right.
$$

Moreover, we have the following result.

Theorem 3: If the angle separations between the interferers and the desired signal satisfy that $\left|u_{i}-u_{1}\right| \geq 1 / 3$ or $\left|v_{i}-v_{1}\right| \geq$ $1 / 3$ for $i=2,3, \ldots, P$, it can be shown that

$$
E\left\{\widehat{\operatorname{SINR}}_{o}\right\}>\operatorname{SINR}_{o}\left(1-(P-1)\left(\sigma_{c}+\sigma_{r}\right)^{2} / L\right)
$$

where $\sigma_{c}^{2}$ and $\sigma_{r}^{2}$ are given by (A-11).

Proof: Please see Appendix D.
Theorem 3 provides a lower bound of the convergence rate for the proposed 2-D EIC under the situation considered. For example, consider the situation where the direction angle of the desired signal $\left(u_{1}, v_{1}\right)=(0,0)$, the blocking orders $(\beta, \delta)=(1,1)$, and the direction angles of interferers $\left(u_{i}, v_{i}\right)$ with $\left|u_{i}\right| \geq 1 / 3$ or $\left|v_{i}\right| \geq 1 / 3$ for $i=2,3, \ldots, P$. Then, we have $\sigma_{c}^{2}=\sigma_{r}^{2}=4$. A lower bound of the output SINR can be obtained from (34) and is given by

$$
E\left\{\widehat{\operatorname{SINR}}_{o}\right\}>\operatorname{SINR}_{o}(1-16(P-1) / L) .
$$

Equation (35) shows that a satisfactory convergence speed for the designed 2-D EIC can be guaranteed in this case.

To result in a simpler version of the above theoretical results for providing an insight, we next consider a special situation where all the uncorrelated interferers are located outside the array mainlobe and the angle separations between the desired signal and the interferers are large enough so that

$$
A_{I}\left(A_{I}^{H} A_{I}\right)^{-1} A_{I}^{H} A\left(u_{1}, v_{1}\right) \ll A\left(u_{1}, v_{1}\right) .
$$

Moreover, $\bar{M}$ and $\bar{N}$ are greater than $2 \beta$ and $2 \delta$, respectively. Based on these two conditions, the optimal weight vector given by (17) can be reduced to an approximation of $W_{o} \approx A\left(u_{1}, v_{1}\right)$ and, hence, $\omega \approx M N$, the results in (31) can be simplified as the following approximations:

$$
\left\{\begin{aligned}
\omega_{c, c} \approx & 2 N \sum_{i=0}^{\beta-1}\left[\sum_{k=0}^{i}(-1)^{k}\left(\begin{array}{l}
\beta \\
k
\end{array}\right)\right]^{2}, \\
\omega_{r, r} \approx & 2 M \sum_{i=0}^{\delta-1}\left[\sum_{k=0}^{i}(-1)^{k}\left(\begin{array}{l}
\delta \\
k
\end{array}\right)\right]^{2} \\
\omega_{c, r}= & \omega_{r, c}^{*} \approx e^{j\left(\pi u_{1}-\pi v_{1}\right)}, \\
& \text { for }(\beta, \delta)=(1,1), \text { and } \approx 0, \quad \text { for }(\beta, \delta) \neq(1,1) .
\end{aligned}\right.
$$

Then, we can simply substitute (33) and (37) into (30) to obtain the corresponding FSP.

\section{Performance Analysis UNDER STEERING ANGLE ERROR}

In this case, the steering angle is not accordant with the direction angle of the desired signal, i.e., $\left(u_{0}, v_{0}\right) \neq\left(u_{1}, v_{1}\right)$. The blocking factors shown in (6) and (7) become

$$
d_{c, i}=\left(e^{j \pi u_{i}}-e^{j \pi u_{0}}\right)^{\beta} \quad \text { and } \quad d_{r, i}=\left(e^{j \pi v_{i}}-e^{j \pi v_{0}}\right)^{\delta}
$$

respectively. The mismatch between $\left(u_{0}, v_{0}\right)$ and $\left(u_{1}, v_{1}\right)$ leads to a result that the blocked data correlation matrix $R$ given by (8) contains a leakage due to the desired signal and becomes

$$
R=A_{S} \Psi_{S} A_{S}^{H}+\pi_{n} \Phi
$$

where $A_{S}=\left[A\left(u_{1}, v_{1}\right), \ldots, A\left(u_{P}, v_{P}\right)\right], \Psi_{S}=\tilde{D}_{c} \bar{\Psi}_{S} \tilde{D}_{c}^{H}+$ $\tilde{D}_{r} \bar{\Psi}_{S} \tilde{D}_{r}^{H}, \tilde{D}_{c}=\operatorname{diag}\left\{d_{c, 1}, \ldots, d_{c, P}\right\}$, and $\tilde{D}_{r}=$ $\operatorname{diag}\left\{d_{r, 1}, \ldots, d_{r, P}\right\}$, respectively. Since $\left(d_{c, i}, d_{r, i}\right) \neq(0,0)$ for all $i=1,2, \ldots, P, \Psi_{S}$ is a $P \times P$ positive definite matrix. Hence the matrix $R_{w}=R+\pi_{n} \Omega$ has $P$ principal eigenvalues 
which are greater than $\sigma^{2} \pi_{n}$ and the corresponding eigenvectors spans the subspace range $\left\{A_{S}\right\}$. The computed basis matrix $E_{I}$ will contain more than $P-1$ principal eigenvectors of $R_{w}$ if the number of interferers is overestimated. From (23), the optimal weight vector corresponding to this case is given by

$$
W_{o}=\left(I_{M N}-E_{I} E_{I}^{H}\right) A\left(u_{0}, v_{0}\right) .
$$

This leads to the result that the 2-D EIC fails to work due to that range $\left\{E_{I}\right\}$ contains the vector $A\left(u_{1}, v_{1}\right)$ and the constraint of $W^{H} E_{I}=0$.

Next, consider the situation where the number of interferers is exactly known and the desired signal is uncorrelated with the $(P-1)$ interferers. Based on (39), we have

$$
R_{w}=R+\pi_{n} \Omega=\pi_{1} A\left(u_{1}, v_{1}\right) A\left(u_{1}, v_{1}\right)^{H}+R_{I}+\sigma^{2} \pi_{n} I_{M N}
$$

where $\pi_{1}=\left(\left|d_{c, 1}\right|^{2}+\left|d_{r, 1}\right|^{2}\right) E\left\{\left|\bar{s}_{1}(t)\right|^{2}\right\}$ denotes the power of the desired signal leakage in the output after the 2-D blocking operation. $R_{I}=A_{I} \Psi_{I} A_{I}$, where $\Psi_{I}$ is given by (14) except that the entries of $D_{c}$ and $D_{r}$ are now given by (38). Let the $P-1$ nonzero eigenvalues and the corresponding eigenvectors of $R_{I}$ be given by $\kappa_{1} \geq \cdots \geq \kappa_{P-1}>0$ and $z_{i}$ for $i=1,2, \ldots, P-1$, respectively. For further simplicity, assume that the interferers are located far away from the desired signal so that $A\left(u_{1}, v_{1}\right)^{H} A\left(u_{i}, v_{i}\right) \approx 0$ for $i=2,3, \ldots, P$. Then, the eigenvalues $\lambda_{i}$ which are greater than $\sigma^{2} \pi_{n}$ and the corresponding eigenvectors $e_{i}$ of $R_{w}$ can be approximated as $\lambda_{i} \approx \kappa_{i}+\sigma^{2} \pi_{n}$ and $e_{i} \approx z_{i}$ for $i=1,2, \ldots, P-1, \lambda_{P} \approx$ $M N \pi_{1}+\sigma^{2} \pi_{n}$ and $e_{P} \approx A\left(u_{1}, v_{1}\right) / \sqrt{M N}$, respectively. Note that $E_{I}$ consists of the first $P-1$ principal eigenvectors of $R_{w}$. As a result, $E_{I}$ consists of $z_{i}$ for $i=1,2, \ldots, P-1$ when $M N \pi_{1}<\kappa_{P-1}$. Hence, $\operatorname{range}\left\{E_{I}\right\} \approx \operatorname{range}\left\{A_{I}\right\}$ and the 2-D EIC works normally. On the other hand, $E_{I}$ contains the normalized response vector $A\left(u_{1}, v_{1}\right) / \sqrt{M N}$ if $M N \pi_{1}>\kappa_{P-1}$. From the optimal weight vector given by (40), we note that the desired signal will be suppressed due to the constraint of $W_{O}^{H} E_{I}=0$. As shown by (38) and the fact that $\pi_{1}$ is proportional to $\left(\left|d_{c, 1}\right|^{2}+\left|d_{r, 1}\right|^{2}\right)$, this difficulty could be alleviated by increasing the orders $\beta$ and $\delta$ if the steering angle error is small. In general, the breakdown threshold $M N \pi_{1}>\kappa_{P-1}$ happens when $R_{I}$ is nearly rank-deficient. To look into the effect of $M N \pi_{1}>\kappa_{P-1}$, we proceed to consider the case of two uncorrelated and closely separated interferers.

Let the two uncorrelated interferers be closely separated so that $A\left(u_{2}, v_{2}\right)^{H} A\left(u_{3}, v_{3}\right) \approx M N$. From [8, pp. 25-27], we can easily show that

$$
\kappa_{2}=\frac{M N \pi_{2} \pi_{3}}{\pi_{2}+\pi_{3}}\left(1-\left|g_{c, 2,3}\right|^{2}\left|g_{r, 2,3}\right|^{2}\right) .
$$

where $\pi_{i}=\left(\left|d_{c, i}\right|^{2}+\left|d_{r, i}\right|^{2}\right) E\left\{\left|\bar{s}_{i}(t)\right|^{2}\right\}$ for $i=2$ and 3 . $g_{c, 2,3}$ and $g_{r, 2,3}$ are given by

$$
\left\{\begin{array}{l}
g_{c, 2,3}=\frac{\sin \left(\pi M\left(u_{2}-u_{3}\right) / 2\right)}{M \sin \left(\pi\left(u_{2}-u_{3}\right) / 2\right.} e^{j \pi(M-1)\left(u_{2}-u_{3}\right) / 2} \\
g_{r, 2,3}=\frac{\sin \left(\pi N\left(v_{2}-v_{3}\right) / 2\right)}{N \sin \left(\pi\left(v_{2}-v_{3}\right) / 2\right)} e^{j \pi(N-1)\left(v_{2}-v_{3}\right) / 2} .
\end{array}\right.
$$

Hence, the condition $M N \pi_{1}>\kappa_{2}$ causing the performance failure becomes

$$
1-\left|g_{c, 2,3}\right|^{2}\left|g_{r, 2,3}\right|^{2}<\pi_{1}\left(\pi_{2}^{-1}+\pi_{3}^{-1}\right) .
$$

When $\left|u_{2}-u_{3}\right|$ and $\left|v_{2}-v_{3}\right|$ are small enough, it is also shown in [8] that

$$
\left\{\begin{array}{l}
\left|g_{c, 2,3}\right|^{2} \approx 1-\frac{M^{2}-1}{12} \pi^{2}\left(u_{2}-u_{3}\right)^{2} \\
\left|g_{r, 2,3}\right|^{2} \approx 1-\frac{N^{2}-1}{12} \pi^{2}\left(v_{2}-v_{3}\right)^{2} .
\end{array}\right.
$$

Substituting (44) into (43) and taking the first-order approximation yields the following performance breakdown threshold

$$
\begin{aligned}
& \frac{M^{2}-1}{12} \pi^{2}\left(u_{2}-u_{3}\right)^{2}+\frac{N^{2}-1}{12} \pi^{2}\left(v_{2}-v_{3}\right)^{2} \\
& \quad=\pi_{1}\left(\pi_{2}^{-1}+\pi_{3}^{-1}\right) .
\end{aligned}
$$

\section{COMPUTER Simulation EXAMPLES}

In this section, several simulation examples for illustrating and confirming the theoretical works are presented. The 2-D array used for all simulations is a URA with $\bar{M}=7$ and $\bar{N}=6$. Moreover, the simulation results based on the direct GEVD of $R$ given by (18) to obtain an IS basis matrix required for computing the optimal weight vector are also presented for comparison.

Example 1: This example is performed to illustrate the theoretical results presented in Section III. We set $(\beta, \delta)=$ $(1,1)$. The desired signal with input $\mathrm{SNR}=0 \mathrm{~dB}$ is impinging on the array from $\left(u_{1}, v_{1}\right)=(0,0)$. One interferer has input $\mathrm{INR}=20 \mathrm{~dB}$. The first $K=50$ data snapshots are used to estimate the source number $P$ and the noise power $\pi_{n}$. Fig. 1 plots the array output SINR in $\mathrm{dB}$ versus the number of snapshots $L$ for two different interfering angles. Each simulation result is obtained by averaging 100 independent runs with independent noise samples for each run. The solid curve represents the theoretical results computed by using (29) based on (28) and (30)-(32). This confirms the validity of (29) given by Theorem 2. On the other hand, the curve with " $x$ " represents the simulation results for the performance of the 2-D EIC designed by using the proposed technique, while the curve with "o" represents the simulation results of the 2D EIC designed by using the direct GEVD technique. The coincidence between these two curves shows that the 2-D EIC designed by using the proposed technique provides the same performance as that directly using the complicated GEVD technique.

Comparing the results of Fig. 1(a) and (b), we note that the output SINR of Fig. 1(a) is smaller than that of Fig. 1(b) for each number of snapshots as expected because the angle separation between the desired signal and the interferer is smaller for Fig. 1(a). This phenomenon is further demonstrated in Fig. 2 by plotting the $F S P$ of (30) versus the interfering angle $\left(u_{2}, v_{2}\right)$. We note that FSP increases and hence the performance degradation increases as $\left(u_{2}, v_{2}\right)$ approaches $\left(u_{1}, v_{1}\right)=(0,0)$. 


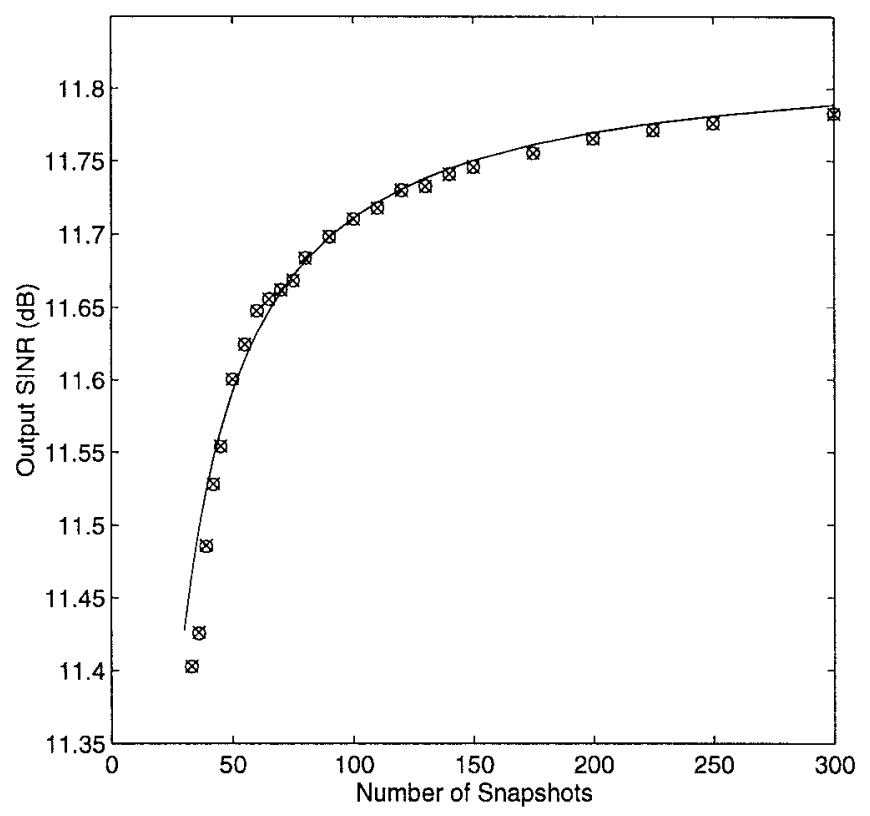

(a)

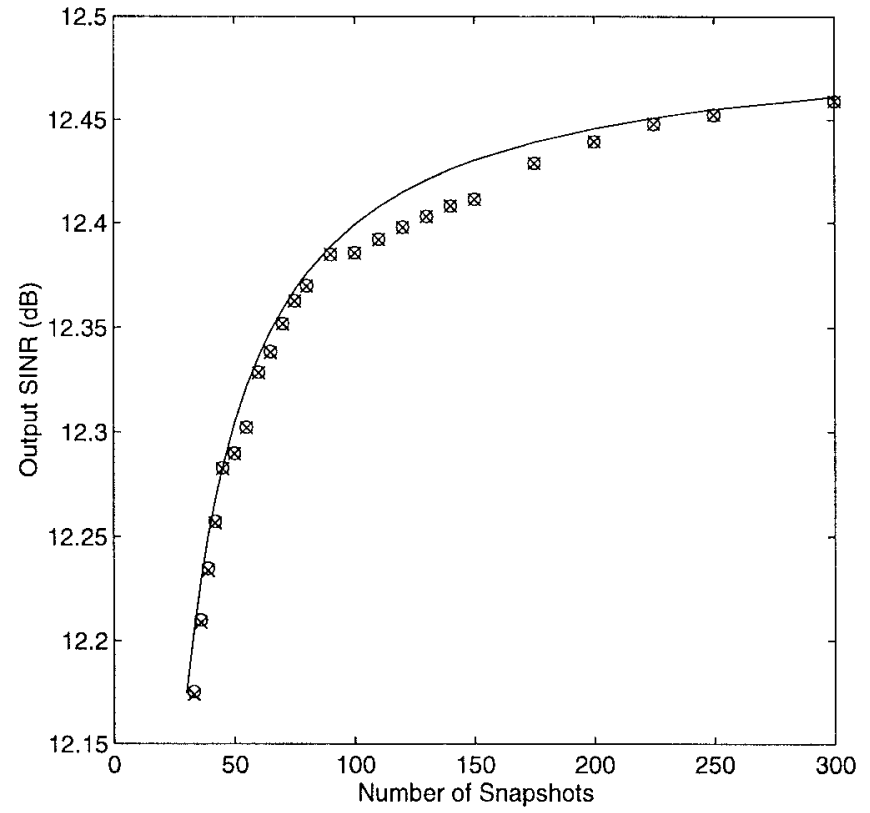

(b)

Fig. 1. The results of Example 1. Output SINR versus the number of snapshots for the case of one interferer. Solid line: The theoretical result. " $x$ ": The 2-D EIC using the proposed technique. "o": The 2-D EIC using the direct GEVD technique. (a) $\left(u_{2}, v_{2}\right)=(0.11,0.13)$. (b) $\left(u_{2}, v_{2}\right)=(0.14,0.12)$.

Example 2: This example considers the case of multiple interferers. Again, we set $(\beta, \delta)=(1,1)$. The desired signal

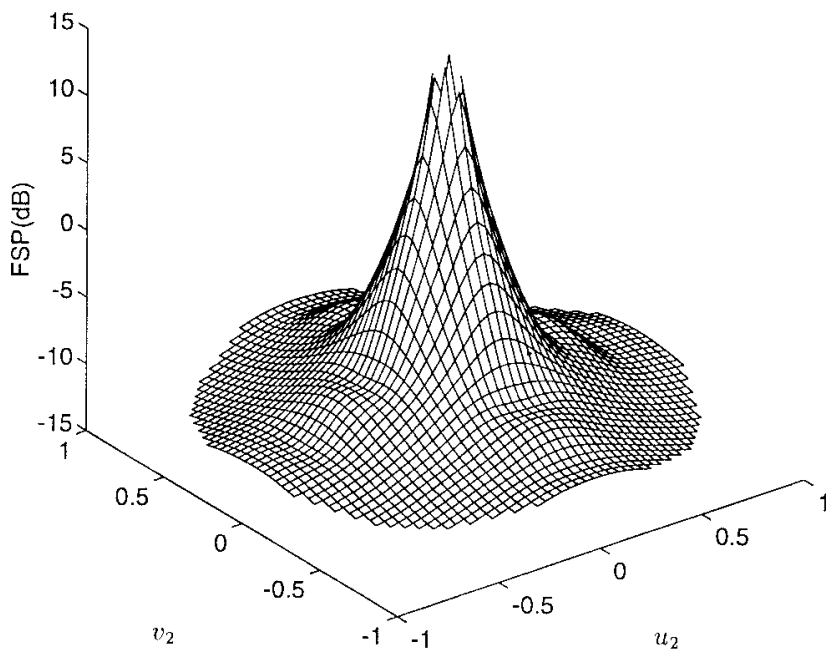

Fig. 2. The factor of statistical performance (FSP) versus the interfering angle for Example 1.

with input $\mathrm{SNR}=0 \mathrm{~dB}$ is impinging on the array from $\left(u_{1}, v_{1}\right)=(0,0)$, while the uncorrelated interferers have the same input $\mathrm{INR}=20 \mathrm{~dB}$. The first $K=50$ data snapshots are used to estimate the source number $P$ and the noise power $\pi_{n}$. Fig. 3 depicts the array output SINR in decibels versus the number of snapshots $L$ for different interfering situations. Each simulation result is obtained by averaging 100 independent runs with independent noise samples for each run. The solid curve represents the theoretical results computed by using (29) based on (28) and (30)-(32). In contrast, the dash curve represents the theoretical results computed by using (29) based on the approximations described by (37). The dash curve almost coincides with the solid curve. This confirms the validity of the approximations given by (37). Moreover, the coincidence between the curves with " $\mathrm{x}$ " and "o" illustrates that the 2-D EIC's designed by using the proposed technique and directly using the complicated GEVD technique have the same performance.

Example 3: Here, we illustrate the performance of the designed 2-D EIC in the presence of steering angle error. The steering angle is $\left(u_{o}, v_{0}\right)=(0,0)$. The desired signal with input $\mathrm{SNR}=3 \mathrm{~dB}$ is impinging on the 2-D array from $\left(u_{1}, v_{1}\right)=(0.03,0.03)$. Two uncorrelated interferers with input $\mathrm{INR}=3 \mathrm{~dB}$ are impinging on the array from $\left(u_{2}, v_{2}\right)=(0.5,0.6)$ and $\left(u_{3}, v_{3}\right)=\left(u_{2}+\Delta u, v_{2}+\Delta v\right)$. To evaluate the sensitivity to the angle separation $(\Delta u, \Delta v)$, we define a robustness index (RI) as follows in (46), shown at the bottom of the page, for the designed 2-D EIC and (47), shown at the bottom of the page, for the 2-D EIC directly

${ }^{R I_{p}}=\frac{\text { The output SINR using } W_{o} \text { of }(40)}{\text { The output SINR using } W_{o} \text { of }(17) \text { with } A\left(u_{1}, v_{1}\right) \text { replaced by } A\left(u_{0}, v_{0}\right)}$

$R I_{h}=\frac{\text { The output SINR using } W_{o} \text { of (19) with } A\left(u_{1}, v_{1}\right) \text { replaced by } A\left(u_{0}, v_{0}\right)}{\text { The output SINR using } W_{o} \text { of (17) with } A\left(u_{1}, v_{1}\right) \text { replaced by } A\left(u_{0}, v_{0}\right)}$ 


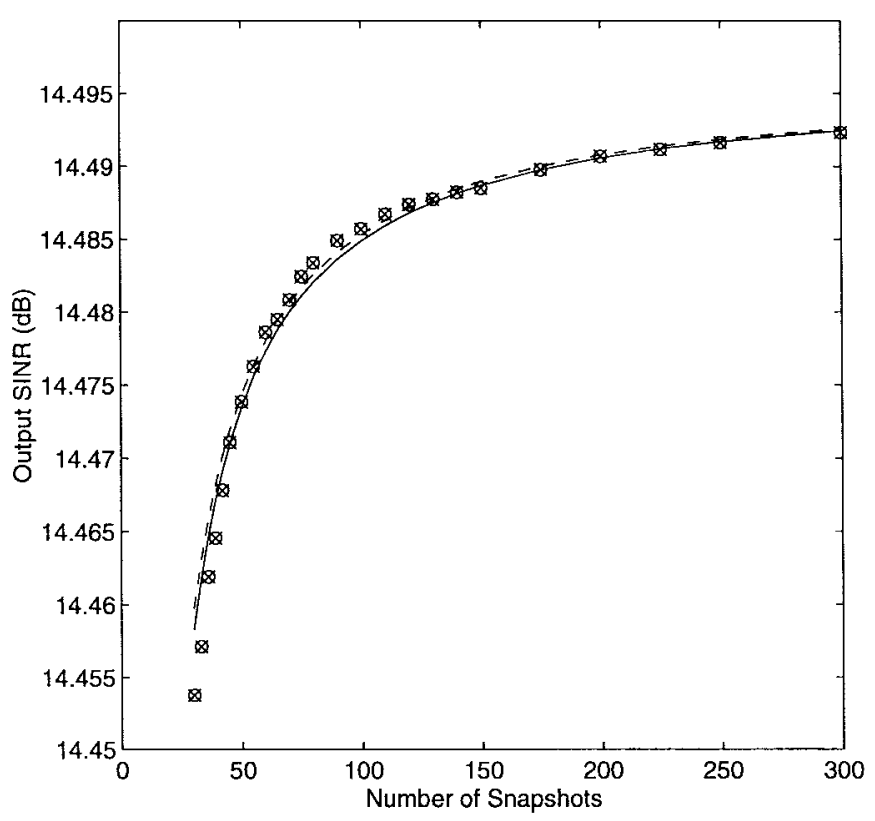

(a)

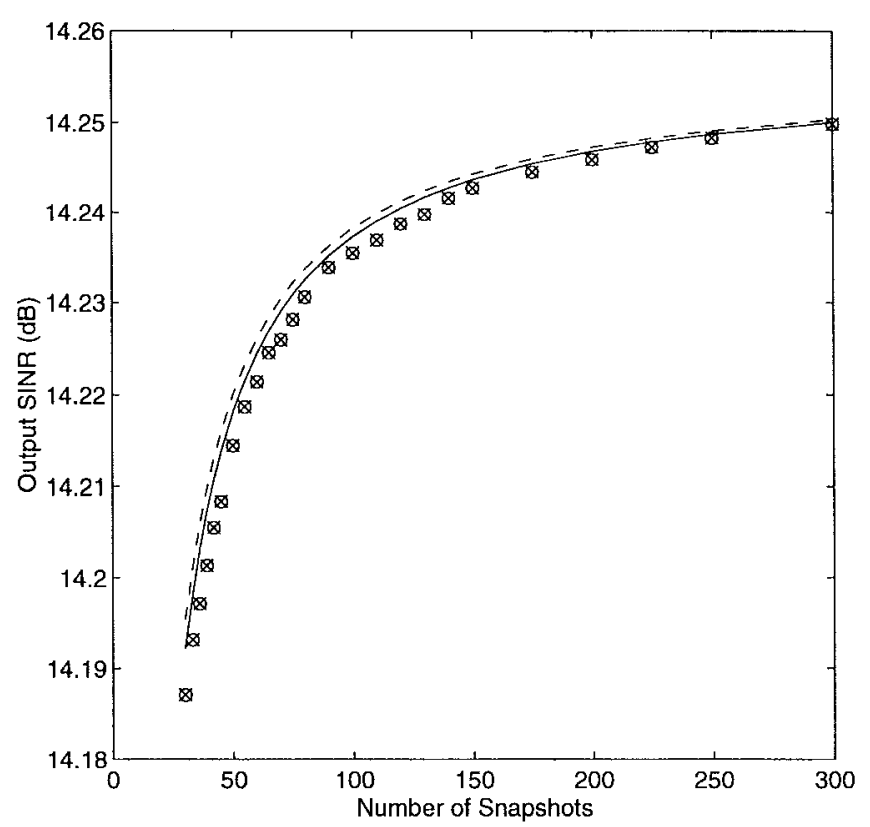

(b)

Fig. 3. The results of Example 2. Output SINR versus the number of snapshots. Solid line and dash line: The theoretical results. " $x$ ": The 2-D EIC using the proposed technique. "o": The 2-D EIC using the direct GEVD technique. (a) Two interferers with $\left(u_{2}, v_{2}\right)=(0,0.6)$ and $\left(u_{3}, v_{3}\right)=(0.55,0.45)$. (b) Three interferers with $\left(u_{2}, v_{2}\right)=(0,0.6),\left(u_{3}, v_{3}\right)=(0.55,0.45)$ and $\left(u_{4}, v_{4}\right)=(0.5,0)$.

using the complicated GEVD technique. Fig. 4(a) plots the $R I$ versus $(\Delta u, \Delta v)$. The top curve represents the $R I_{p}$ versus $(\Delta u, \Delta v)$, while the bottom curve represents the $R I_{h}$ versus $(\Delta u, \Delta v)$. It shows that the proposed technique possesses the advantage of robust capability against steering angle error over the GEVD technique. Fig. 4(b) depicts the curves of the breakdown threshold for $R I_{p}=0.5$. The dash curve represents the breakdown threshold computed by (45), while the solid curve represents the simulation results. This figure also confirms the presented theoretical results.

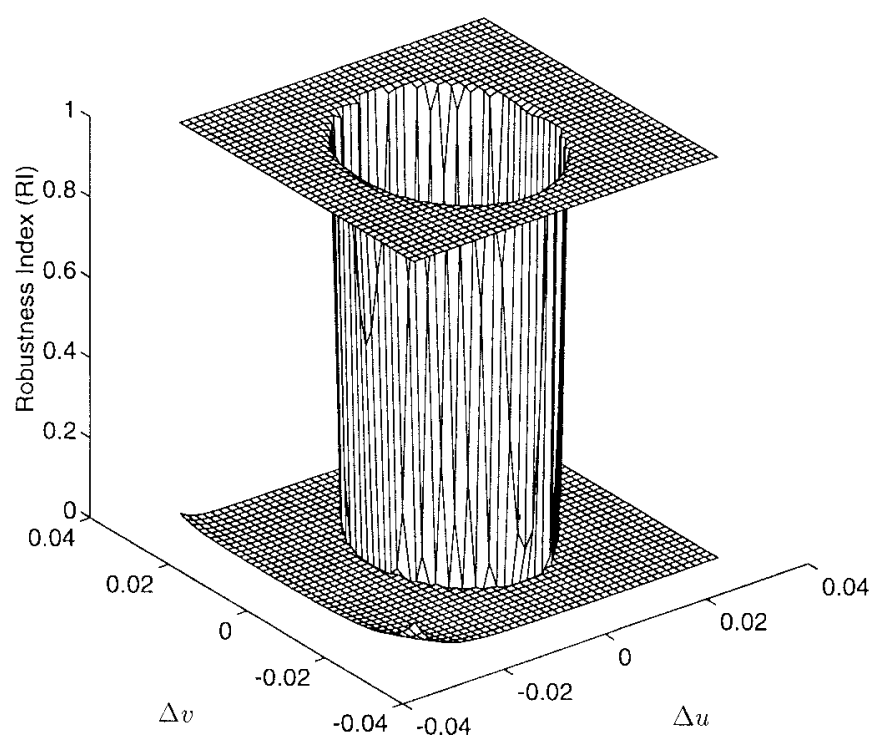

(a)

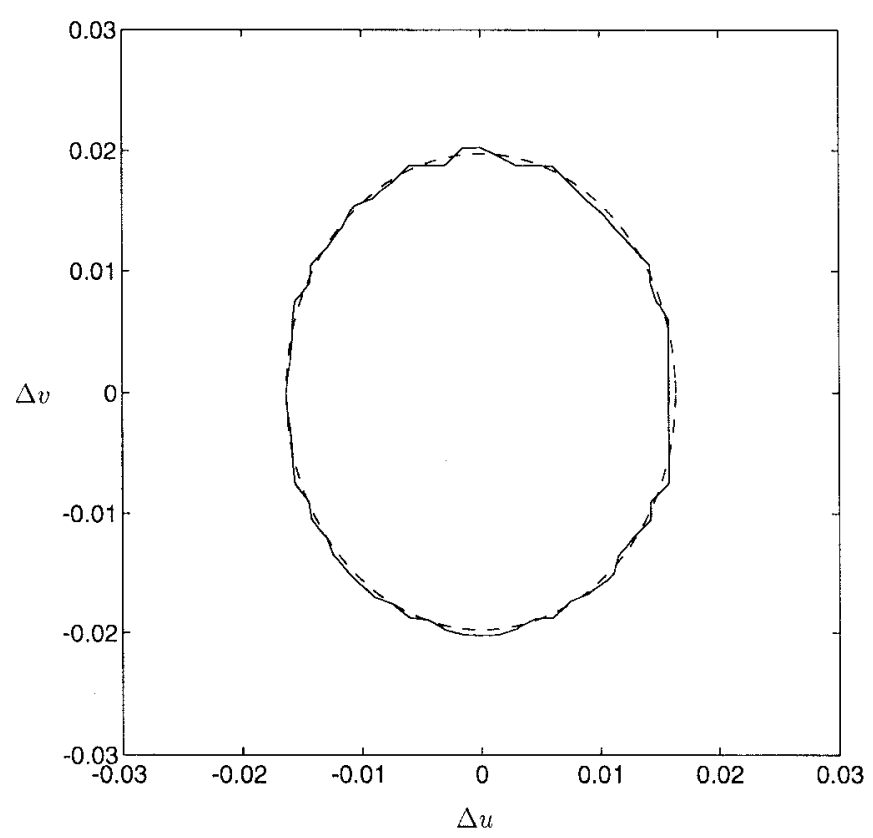

(b)

Fig. 4. The performance comparison for Example 3. (a) Robustness index versus the interfering angle separation $(\Delta u, \Delta v)$. The top curve: The 2-D EIC using the proposed technique. The bottom curve: The 2-D EIC using the direct GEVD technique. (b) The breakdown threshold curves of the 2-D EIC using the proposed technique. Solid curve: The simulation results. Dash curve: The theoretical results.

\section{CONCLUSION}

The theoretical works for the design and analysis of a 2-D eigenspace-based interference canceller (EIC) have been presented. An effective 2-D signal blocking technique is first presented to remove the desired signal from the received array data. To compensate the effect of the signal blocking operation on the sensor noise, a positive definite matrix has been constructed. Therefore, the interference subspace required for computing the optimal weight vector can be obtained by using conventional eigenvalue decomposition methods. The performances of the designed 2-D EIC under finite samples 
and steering angle error have been evaluated, respectively. The developed theoretical results are confirmed by several simulation examples. It has been shown that the performance of the designed 2-D EIC is the same as that of a 2-D EIC directly using a complicated GEVD technique in the situation without steering angle error. However, the proposed 2-D EIC possesses the advantage of more robust capability against steering angle error over the 2-D EIC based on the GEVD technique.

\section{APPENDIX A}

Let $\tilde{I}_{m}$ be an $m \times m$ cyclic-shifting matrix defined as

$$
\tilde{I}_{m}=\left[\begin{array}{lllll}
I_{m, 2} & I_{m, 3} & \cdots & I_{m, m} & I_{m, 1}
\end{array}\right]
$$

where $I_{m, i}$ is the $i$ th column vector of the $m \times m$ identity matrix. Following the results presented in [4], the blocking matrix $B_{c}$ which satisfies (6) can be constructed as follows:

$$
B_{c}=\left[\begin{array}{lllll}
\tilde{b}_{c} & \tilde{I}_{\bar{M}} \tilde{b}_{c} & \cdots & \left(\tilde{I}_{\bar{M}}\right)^{M-1} \tilde{b}_{c}
\end{array}\right]
$$

where $\tilde{b}_{c}$ is an $\bar{M} \times 1$ vector given by

$$
\tilde{b}_{c}=\left[b_{c, 0}, \ldots, b_{c, \beta}, 0, \ldots, 0\right]^{T}
$$

and $b_{c, k}$ are the coefficients satisfying

$$
\left(z-e^{j \pi u_{1}}\right)^{\beta}=\sum_{k=0}^{\beta} b_{c, k}^{*} z^{k} .
$$

The subscript “*” denotes the complex conjugate. From (A.2) and (A.3), it can be seen that $B_{c}^{H} B_{c}$ is an $M \times M$ Hermitian and Toeplitz matrix. Furthermore, let $H T\left\{x_{1}, x_{2}, \ldots, x_{m}\right\}$ denote an $m \times m$ Hermitian and Toeplitz matrix with its first row given by $\left[x_{1}, x_{2}, \ldots, x_{m}\right]$. Then, we have

$$
\begin{aligned}
B_{c}^{H} B_{c}=H T\left\{\epsilon_{c, 0}, \epsilon_{c, 1}, \ldots, \epsilon_{c, M-1}\right\} \\
\text { with } \epsilon_{c, i}=\sum_{k=0}^{\beta-i} b_{c, k+i}^{*} b_{c, k} .
\end{aligned}
$$

Next, we construct an $(M+i) \times 1$ vector as follows:

$$
f_{c}(k, i)=\left[\begin{array}{llll}
1 & O_{1, i-1} & j^{k} & O_{1, M-1}
\end{array}\right]^{T}
$$

where $i=1,2, \ldots, M-1$ and $k$ is an integer. From (A.6), an $(M+i) \times M$ matrix is constructed as follows:

$$
\begin{aligned}
& F_{c}(k, i)=\left[\begin{array}{llll}
f_{c}(k, i) & \tilde{I}_{M+i} f_{c}(k, i) & \tilde{I}_{M+i}^{2} f_{c}(k, i) & \cdots
\end{array}\right. \\
& \left.\tilde{I}_{M+i}^{M-1} f_{c}(k, i)\right] \text {. }
\end{aligned}
$$

Using (A.6) and (A.7), we have

$$
\begin{aligned}
\Gamma_{c}(k, i) & =F_{c}(k, i)^{H} F_{c}(k, i) \\
& =H T\left\{2, O_{1, i-1},(-j)^{k}, O_{M-1-i}\right\} .
\end{aligned}
$$

From (A.8), we note that $\Gamma_{c}(k, i)$ is positive definite, Hermitian, and Toeplitz. Moreover, it is easy to show that

$$
\begin{gathered}
\left|\operatorname{Re}\left\{\epsilon_{c, i}\right\}\right| \Gamma_{c}\left(2 \operatorname{sgn}\left(\operatorname{Re}\left\{\epsilon_{c, i}\right\}\right), i\right) \\
+\left|\operatorname{Im}\left\{\epsilon_{c, i}\right\}\right| \Gamma_{c}\left(2 \operatorname{sgn}\left(\operatorname{Im}\left\{\epsilon_{c, i}\right\}\right)-1, i\right) \\
=H T\left\{2\left(\left|\operatorname{Re}\left\{\epsilon_{c, i}\right\}\right|+\left|\operatorname{Im}\left\{\epsilon_{c, i}\right\}\right|\right),\right. \\
\left.O_{1, i-1},-\epsilon_{c, i}, O_{1, M-1-i}\right\}
\end{gathered}
$$

for $i=1,2, \ldots, M-1$, where $\operatorname{Re}\{x\}$ and $\operatorname{Im}\{x\}$ denote the real and imaginary parts of $x$, respectively. $\operatorname{sgn}(x)=1$ if $x \geq 0$, and $=0$, otherwise. We then construct a positive definite matrix as follows:

$$
\begin{aligned}
\Omega_{c}= & \sum_{i=1}^{M-1}\left(\left|\operatorname{Re}\left\{\epsilon_{c, i}\right\}\right| \Gamma_{c}\left(2 \operatorname{sgn}\left(\operatorname{Re}\left\{\epsilon_{c, i}\right\}\right), i\right)\right. \\
& \left.+\left|\operatorname{Im}\left\{\epsilon_{c, i}\right\}\right| \Gamma_{c}\left(2 \operatorname{sgn}\left(\operatorname{Im}\left\{\epsilon_{c, i}\right\}\right)-1, i\right)\right) .
\end{aligned}
$$

Summing (A.5) and (A.10) thus yields a diagonal matrix as follows:

$$
\begin{aligned}
B_{c}^{H} B_{c}+\Omega_{c} & =\left(\epsilon_{c, 0}+2 \sum_{i=1}^{M-1}\left(\left|\operatorname{Re}\left\{\epsilon_{c, i}\right\}\right|+\left|\operatorname{Im}\left\{\epsilon_{c, i}\right\}\right|\right)\right) I_{M} \\
& =\sigma_{c}^{2} I_{M}
\end{aligned}
$$

where $\sigma_{c}^{2}$ denotes the proportional constant. Following the same procedure, we can find a positive definite matrix $\Omega_{r}$ such that $B_{r}^{H} B_{r}+\Omega_{r}=\sigma_{r}^{2} I_{N}$ for some positive $\sigma_{r}^{2}$. Finally, we form the following matrix:

$$
\Omega=I_{N} \otimes \Omega_{c}+\Omega_{r} \otimes I_{M}
$$

Based on (15) and the property of Kronecker product [7]

$$
\langle\mathrm{KP} .3\rangle \quad\left(\sum_{i} Q_{i}\right) \otimes\left(\sum_{k} T_{k}\right)=\sum_{i} \sum_{k}\left(Q_{i} \otimes T_{k}\right)
$$

for matrices $Q_{i}$ and $T_{k}$ with appropriate sizes, we can easily show that $\Phi+\Omega=\sigma^{2} I_{M N}$, where $\sigma^{2}=\sigma_{c}^{2}+\sigma_{r}^{2}$.

\section{APPENDIX B}

Here, we show the result given by (29) in Theorem 2. Performing the EVD of $R_{w}$, we obtain the following expression:

$$
R_{w}=E_{I} \Lambda_{I} E_{I}^{H}+E_{R} \Lambda_{R} E_{R}^{H}
$$

where $\Lambda_{I}=\operatorname{diag}\left\{\lambda_{1}, \lambda_{2}, \ldots, \lambda_{P-1}\right\}$ and $\Lambda_{R}=\operatorname{diag}\left\{\lambda_{P}\right.$, $\left.\lambda_{P+1}, \ldots, \lambda_{M N}\right\}=\sigma^{2} \pi_{n} I_{M N}$. Similarly, we have the following expression:

$$
\hat{R}_{w}=\hat{E}_{I} \hat{\Lambda}_{I} \hat{E}_{I}^{H}+\hat{E}_{R} \hat{\Lambda}_{R} \hat{E}_{R}^{H}
$$

from the EVD of $\hat{R}_{w}$, where $\hat{\Lambda}_{I}=\operatorname{diag}\left\{\hat{\lambda}_{1}, \hat{\lambda}_{2}, \ldots, \hat{\lambda}_{P-1}\right\}$ and $\hat{\Lambda}_{R}=\operatorname{diag}\left\{\hat{\lambda}_{P}, \hat{\lambda}_{P+1}, \ldots, \hat{\lambda}_{M N}\right\}$. From (20) and (25), the deviation between $\hat{R}_{w}$ and $R_{w}$ due to finite sample effect can be expressed as

$$
\Delta R_{w}=\hat{R}_{w}-R_{w}=\Delta R+\Delta \pi_{n} \Omega
$$

where $\Delta \pi_{n}=\hat{\pi}_{n}-\pi_{n}$ and $\Delta R=\hat{R}-R$. Using (8) and (26), $\Delta R$ is given by

$$
\Delta R=\tilde{B}_{c}^{H} \Delta \bar{R} \tilde{B}_{c}+\tilde{B}_{r}^{H} \Delta \bar{R} \tilde{B}_{r} .
$$

Following the first-order perturbation analysis presented in [9], we can show that

$$
\Delta E_{R}=\hat{E}_{R}-E_{R} \approx-R_{I}^{+} \Delta R_{w} E_{R}
$$

where

$$
R_{I}^{+}=E_{I}\left(\Lambda_{I}-\sigma^{2} \pi_{n} I_{P-1}\right)^{-1} E_{I}^{H}
$$


It follows from (B-6) that $R_{I}^{+}$possesses the following properties:

$$
\begin{aligned}
R_{I}^{+} & =E_{I}\left(A_{I}^{H} E_{I}\right)^{-1} \Psi_{I}^{-1}\left(A_{I}^{H} E_{I}\right)^{-H} E_{I}^{H}, \quad \text { and } \\
R_{I}^{+} R_{I} R_{I}^{+} & =R_{I}^{+}
\end{aligned}
$$

Substituting (B.5) into (28) and preserving only the first-order term, we obtain the following approximation for the optimal weight vector under finite samples:

$$
\hat{W}_{o} \approx W_{o}+\left(E_{R} \Delta E_{R}^{H}+\Delta E_{R} E_{R}^{H}\right) A\left(u_{1}, v_{1}\right) .
$$

Using (B-8) and the property that $\Delta E_{R}^{H} E_{R}=0$, we can find the powers of the desired signal, the noise, and the interferers at the array output as follows:

$$
\left\{\begin{aligned}
\hat{p}_{s}= & \bar{\pi}_{1}\left|\hat{W}_{o}^{H} A\left(u_{1}, v_{1}\right)\right|^{2} \approx p_{s}+\sum_{k=1}^{3} \Delta p_{s, k} \\
& + \text { the first-order terms } \\
\hat{p}_{n}= & \pi_{n} \hat{W}_{o}^{H} \hat{W}_{o} \approx p_{n}+\sum_{k=1}^{2} \Delta p_{n, k} \\
& + \text { the first-order terms } \\
\hat{p}_{i}= & \hat{W}_{o}^{H} A_{I} \bar{\Psi}_{I} A_{I} \hat{W}_{o} \approx p_{i}+\Delta p_{i} \\
& + \text { the first-order terms }
\end{aligned}\right.
$$

where $p_{s}=\bar{\pi}_{1}\left|W_{o}^{H} A\left(u_{1}, v_{1}\right)\right|^{2}, p_{n}=\pi_{n} W_{o}^{H} W_{o}$, and $p_{i}=$ $W_{o}^{H} A_{I} \bar{\Psi}_{I} A_{I}^{H} W_{o}$ represent the output powers of the desired signal, the noise, and the interferers without finite sample effect, respectively. $\bar{\pi}_{1}=E\left\{\left|\bar{s}_{1}(t)\right|^{2}\right\}$ denotes the input power of the desired signal. $p_{i}$ is negligible when the 2-D EIC works normally. The other terms are the second-order perturbation terms which are given by

$$
\begin{gathered}
\left\{\begin{array}{l}
\Delta p_{s, 1}=\Delta p_{s, 2}^{*}=\bar{\pi}_{1}\left(A\left(u_{1}, v_{1}\right)^{H} E_{R} \Delta E_{R}^{H} A\left(u_{1}, v_{1}\right)\right)^{2} \\
\Delta p_{s, 3}=2 \bar{\pi}_{1}\left|A\left(u_{1}, v_{1}\right)^{H} E_{R} \Delta E_{R}^{H} A\left(u_{1}, v_{1}\right)\right|^{2}
\end{array}\right. \\
\left\{\begin{array}{l}
\Delta p_{n, 1}=\pi_{n} A\left(u_{1}, v_{1}\right)^{H} \Delta E_{R} \Delta E_{R}^{H} A\left(u_{1}, v_{1}\right), \\
\Delta p_{n, 2}=\pi_{n} A\left(u_{1}, v_{1}\right)^{H} E_{R} \Delta E_{R}^{H} \Delta E_{R} E_{R}^{H} A\left(u_{1}, v_{1}\right)
\end{array}\right.
\end{gathered}
$$

and

$$
\Delta p_{i}=A\left(u_{1}, v_{1}\right)^{H} E_{R} \Delta E_{R}^{H} A_{I} \bar{\Psi}_{I} A_{I}^{H} \Delta E_{R} E_{R}^{H} A\left(u_{1}, v_{1}\right)
$$

respectively. Since $p_{i}$ is negligible, the output SINR of the 2-D EIC can be written as

$$
\widehat{\operatorname{SINR}}_{o}=\frac{\hat{p}_{s}}{\hat{p}_{i}+\hat{p}_{n}}=\frac{p_{s}\left(1+\left(\hat{p}_{s}-p_{s}\right) / p_{s}\right)}{p_{n}\left(1+\left(\hat{p}_{n}-p_{n}\right) / p_{n}+\hat{p}_{i} / p_{n}\right)} .
$$

Consider the situation where the number of data snapshots is large enough. Utilizing the first-order approximation of $(1+x)^{-1} \approx 1-x$ for a small $x$, we can obtain an approximation for (B-13) as follows:

$\widehat{\operatorname{SINR}}_{o} \approx \operatorname{SINR}_{o}\left(1+\left(\hat{p}_{s}-p_{s}\right) / p_{s}-\left(\hat{p}_{n}-p_{n}\right) / p_{n}-\hat{p}_{i} / p_{n}\right)$, where $\operatorname{SINR}_{o}=p_{s} / p_{n}$ represents the output SINR without finite sample effect. Since the expectation for each of the firstorder terms in (B-9) is zero, the expectation of the output SINR can be approximated from (B-14) as follows:

$E\left\{\widehat{\operatorname{SINR}}_{o}\right\} \approx \operatorname{SINR}_{o}\left(1+\frac{E\left\{\Delta p_{s}\right\}}{p_{s}}-\frac{E\left\{\Delta p_{n}\right\}}{p_{n}}-\frac{E\left\{\Delta p_{i}\right\}}{p_{n}}\right)$

where $\Delta p_{s}=\sum_{k=1}^{3} \Delta p_{s, k}$ and $\Delta p_{n}=\sum_{k=1}^{2} \Delta p_{n, k}$.

Next, we compute the individual terms in (B.15). As shown in (B.3), the deviation $\Delta R_{w}$ is composed of two independent terms, i.e., $\Delta \pi_{n}$ and $\Delta \bar{R}$. By using the eigenvalue method of [12] to estimate the noise power, it has been shown that

$$
E\left\{\left|\Delta \pi_{n}\right|^{2}\right\}=\frac{\pi_{n}^{2}}{K(M N-P)}
$$

if $K$ data snapshots are used. On the other hand, it has been shown in [10] that the deviation $\Delta \bar{R}$ due to finite sample effect has zero mean and the second-order statistical property as

$$
E\left\{Q_{1}^{H} \Delta \bar{R} Q_{2} Q_{3}^{H} \Delta \bar{R} Q_{4}\right\}=\frac{1}{L} \operatorname{Tr}\left\{Q_{3}^{H} \bar{R} Q_{2}\right\}\left(Q_{1}^{H} \bar{R} Q_{4}\right)
$$

where $Q_{i}$ are matrices with appropriate sizes. By substituting (B.5) into (B.10)-(B.12) and using the properties of (B.7), (B.16), and (B-17), the individual terms in (B.15) are computed. The results are listed in Appendix C. It is also shown in Appendix $\mathrm{C}$ that the term $L E\left\{\Delta p_{i, a}\right\} / p_{n}$ is dominant in the case of input INR high enough since all the other terms decrease as the input INR increases. Accordingly, (B-15) can be approximately expressed as

$$
E\left\{\widehat{\operatorname{SINR}}_{o}\right\} \approx \operatorname{SINR}_{o}\left(1-\frac{1}{L} F S P\right)
$$

for input INR high enough, where the factor of statistical performance $F S P=L E\left\{\Delta p_{i, a}\right\} / p_{n}$ is given by (30).

\section{APPENDIX C}

To ease the presentation, we employ the subscripts $x(1)$ and $x(2)$ to replace the subscripts " $r$ " and " $c$ ", respectively. For example, $B_{x(1)}$ and $B_{x(2)}$ represent the notations $B_{r}$ and $B_{c}$, respectively. Thus, (B-4) can be rewritten as

$$
\Delta R=\sum_{i=1}^{2} \tilde{B}_{x(i)}^{H} \Delta \bar{R} \tilde{B}_{x(i)} .
$$

Using the above notations and performing some algebraic manipulation provides

$$
\left\{\begin{aligned}
E\left\{\Delta p_{i}\right\} / p_{n} \approx & E\left\{\Delta p_{i, a}\right\} / p_{n}+E\left\{\Delta p_{i, b}\right\} / p_{n} \\
& +E\left\{\Delta p_{i, c}\right\} / p_{n} \\
E\left\{\Delta p_{i, a}\right\} / p_{n}= & (L \omega)^{-1} \sum_{i, k}\left(\xi_{x(k), x(i)} \omega_{x(i), x(k)}\right) \\
E\left\{\Delta p_{i, b}\right\} / p_{n}= & (L \omega)^{-1} \sum_{i, k}\left(\operatorname { T r } \left\{\pi_{n} R_{I}^{+} A_{I} \bar{\Psi}_{I}\right.\right. \\
& \left.\left.\times A_{I}^{H} R_{I}^{+} \tilde{B}_{x(k)}^{H} \tilde{B}_{x(i)}\right\} \omega_{x(i), x(k)}\right) \\
E\left\{\Delta p_{i, c}\right\} / p_{n}= & (K(M N-P) \omega)^{-1} \\
& \times\left(\pi_{n} W_{o}^{H} \Omega R_{I}^{+} A_{I} \bar{\Psi}_{I} A_{I}^{H} R_{I}^{+} \Omega W_{o}\right)
\end{aligned}\right.
$$




$$
\left\{\begin{aligned}
E\left\{\Delta p_{n, 1}\right\} / p_{n} \approx & E\left\{\Delta p_{n, 1 a}\right\} / p_{n} \\
& +E\left\{\Delta p_{n, 1 b}\right\} / p_{n}+E\left\{\Delta p_{n, 1 c}\right\} / p_{n} \\
E\left\{\Delta p_{n, 1 a}\right\} / p_{n}= & (L \omega)^{-1} \sum_{i, k}\left(\operatorname { T r } \left\{E_{R} E_{R}^{H}\right.\right. \\
& \left.\left.\times \tilde{B}_{x(k)}^{H} \tilde{B}_{x(i)}\right\} \eta_{x(i), x(k)}\right) \\
E\left\{\Delta p_{n, 1 b}\right\} / p_{n}= & (L \omega)^{-1} \sum_{i, k}\left(\operatorname { T r } \left\{E_{R} E_{R}^{H}\right.\right. \\
& \left.\left.\times \tilde{B}_{x(k)}^{H} \tilde{B}_{x(i)}\right\} \rho_{x(i), x(k)}\right) \\
E\left\{\Delta p_{n, 1 c}\right\} / p_{n}= & (K(M N-P) \omega)^{-1}\left(\pi_{n}^{2} A\left(u_{1}, v_{1}\right)^{H}\right. \\
& \left.\times R_{I}^{+} \Omega E_{R} E_{R}^{H} \Omega R_{I}^{+} A\left(u_{1}, v_{1}\right)\right)
\end{aligned}\right.
$$

$$
\left\{\begin{aligned}
E\left\{\Delta p_{n, 2}\right\} / p_{n} \approx & E\left\{\Delta p_{n, 2 a}\right\} / p_{n}+E\left\{\Delta p_{n, 2 b}\right\} / p_{n} \\
& +E\left\{\Delta p_{n, 2 c}\right\} / p_{n} \\
E\left\{\Delta p_{n, 2 a}\right\} / p_{n}= & (L \omega)^{-1} \sum_{i, k}\left(\operatorname { T r } \left\{\pi_{n} R_{I}^{+} A_{I}\right.\right. \\
& \left.\left.\times D_{x(k)} \bar{\Psi}_{I} D_{x(i)}^{H} A_{I}^{H} R_{I}^{+}\right\} \omega_{x(i), x(k)}\right) \\
E\left\{\Delta p_{n, 2 b}\right\} / p_{n}= & (L \omega)^{-1} \sum_{i, k}\left(\operatorname { T r } \left\{\pi_{n}^{2} R_{I}^{+} \tilde{B}_{x(k)}^{H}\right.\right. \\
& \left.\left.\times \tilde{B}_{x(i)} R_{I}^{+}\right\} \omega_{x(i), x(k)}\right) \\
E\left\{\Delta p_{n, 2 c}\right\} / p_{n}= & (K(M N-P) \omega)^{-1} \\
& \times\left(\pi_{n}^{2} W_{o}^{H} \Omega R_{I}^{+} R_{I}^{+} \Omega W_{o}\right)
\end{aligned}\right.
$$

$$
\left\{\begin{aligned}
E\left\{\Delta p_{s, 1}\right\} / p_{s} \approx & E\left\{\Delta p_{s, 1 a}\right\} / p_{s}+E\left\{\Delta p_{s, 1 b}\right\} / p_{s} \\
E\left\{\Delta p_{s, 1 a}\right\} / p_{s}= & \left(L \omega^{2}\right)^{-1} \sum_{i, k}\left[\left(\pi_{n} W_{o}^{H}\right.\right. \\
& \left.\times \tilde{B}_{x(i)}^{H} \tilde{B}_{x(k)} R_{I}^{+} A\left(u_{1}, v_{1}\right)\right) \\
& \left.\times\left(\pi_{n} W_{o}^{H} \tilde{B}_{x(k)}^{H} \tilde{B}_{x(i)} R_{I}^{+} A\left(u_{1}, v_{1}\right)\right)\right] \\
E\left\{\Delta p_{s, 1 b}\right\} / p_{s}= & \left(K(M N-P) \omega^{2}\right)^{-1} \\
& \times\left(\pi_{n} W_{o}^{H} \Omega R_{I}^{+} A\left(u_{1}, v_{1}\right)\right)^{2}
\end{aligned}\right.
$$

and

$$
\left\{\begin{aligned}
E\left\{\Delta p_{s, 3}\right\} / p_{s} \approx & E\left\{\Delta p_{s, 3 a}\right\} / p_{s}+E\left\{\Delta p_{s, 3 b}\right\} / p_{s} \\
& +E\left\{\Delta p_{s, 3 c}\right\} / p_{s} \\
E\left\{\Delta p_{s, 3 a}\right\} / p_{s}= & 2\left(L \omega^{2}\right)^{-1} \sum_{i, k}\left(\eta_{x(i), x(k)} \omega_{x(k), x(i)}\right) \\
E\left\{\Delta p_{s, 3 b}\right\} / p_{s}= & 2\left(L \omega^{2}\right)^{-1} \sum_{i, k}\left(\rho_{x(i), x(k)} \omega_{x(k), x(i)}\right) \\
E\left\{\Delta p_{s, 3 c}\right\} / p_{s}= & 2\left(K(M N-P) \omega^{2}\right)^{-1} \\
& \times\left|\pi_{n} W_{o}^{H} \Omega R_{I}^{+} A\left(u_{1}, v_{1}\right)\right|^{2}
\end{aligned}\right.
$$

respectively, where $\omega=W_{o}^{H} W_{o}$ and

$$
\left\{\begin{aligned}
\omega_{x(i), x(k)}= & W_{o}^{H} \tilde{B}_{x(i)}^{H} \tilde{B}_{x(k)} W_{o} \\
\xi_{x(k), x(i)}= & \operatorname{Tr}\left\{\Psi_{I}^{-1} \bar{\Psi}_{I} \Psi_{I}^{-1} D_{x(k)} \bar{\Psi}_{I} D_{x(i)}^{H}\right\} \\
\eta_{x(i), x(k)}= & \pi_{n} A\left(u_{1}, v_{1}\right)^{H} R_{I}^{+} A_{I} D_{x(i)} \bar{\Psi}_{I} D_{x(k)}^{H} \\
& \times A_{I}^{H} R_{I}^{+} A\left(u_{1}, v_{1}\right) \\
\rho_{x(i), x(k)}= & \pi_{n}^{2} A\left(u_{1}, v_{1}\right)^{H} R_{I}^{+} \tilde{B}_{x(i)}^{H} \tilde{B}_{x(k)} R_{I}^{+} A\left(u_{1}, v_{1}\right)
\end{aligned}\right.
$$

for $i, k=1,2$, respectively.

Next, let the positive definite matrix $\bar{\Psi}_{I}$ be expressed as $\bar{\Psi}_{I}=\tilde{a} \bar{\Psi}_{0}$ for some positive number $\tilde{a}$ and positive definite matrix $\bar{\Psi}_{0}$. Then it can be easily shown from (B.7) that $R_{I}^{+}$is proportional to $\tilde{a}^{-1}$. From (C.2) to (C.7), it can also be shown that each of the following terms:

$$
\begin{aligned}
& E\left\{\Delta p_{i, b}\right\} / p_{n}, \quad E\left\{\Delta p_{i, c}\right\} / p_{n}, \quad E\left\{\Delta p_{n, 1 a}\right\} / p_{n}, \\
& E\left\{\Delta p_{n, 2 a}\right\} / p_{n}, \quad \text { and } E\left\{\Delta p_{s, 3 a}\right\} / p_{s}
\end{aligned}
$$

is proportional to $\tilde{a}^{-1}$ and each of the following terms:

$$
\begin{aligned}
& E\left\{\Delta p_{n, 1 b}\right\} / p_{n}, \quad E\left\{\Delta p_{n, 1 c}\right\} / p_{n} \quad E\left\{\Delta p_{n, 2 b}\right\} / p_{n} \\
& E\left\{\Delta p_{n, 2 c}\right\} / p_{n} \quad E\left\{\Delta p_{s, 1 a}\right\} / p_{s} \\
& E\left\{\Delta p_{s, 1 b}\right\} / p_{s} \quad E\left\{\Delta p_{s, 3 b}\right\} / p_{s}, \quad \text { and } \quad E\left\{\Delta p_{s, 3 c}\right\} / p_{s}
\end{aligned}
$$

is proportional to $\tilde{a}^{-2}$, while only the term $E\left\{\Delta p_{i, a}\right\} / p_{n}$ is fixed and independent of $\tilde{a}$. To get a further simplification, consider the case that $\tilde{a}$ is large enough, i.e., the input INR is high enough so that these terms in (C.8) and (C.9) are negligible as compared to $E\left\{\Delta p_{i, a}\right\} / p_{n}$. Then, we have the result as shown by (B-18).

\section{APPENDIX D}

By using the Cauchy-Schwarz inequality that

$$
\left|\operatorname{Tr}\left\{Q_{1} Q_{2}^{H}\right\}\right|^{2} \leq \operatorname{Tr}\left\{Q_{1} Q_{1}^{H}\right\} \operatorname{Tr}\left\{Q_{2} Q_{2}^{H}\right\}
$$

where $Q_{1}$ and $Q_{2}$ are matrices with appropriate sizes, it follows from (31) and (32) that

$$
\left\{\begin{array}{l}
\left|\omega_{c, r}\right|^{2}=\left|\omega_{r, c}\right|^{2} \leq \omega_{c, c} \omega_{r, r} \\
\left|\xi_{c, r}\right|^{2}=\left|\xi_{r, c}\right|^{2} \leq \xi_{c, c} \xi_{r, r}
\end{array}\right.
$$

Based on (30) and (D.2), it can be shown that

$$
F S P \leq\left[\left(\xi_{r, r} \frac{\omega_{r, r}}{\omega}\right)^{1 / 2}+\left(\xi_{c, c} \frac{\omega_{c, c}}{\omega}\right)^{1 / 2}\right]^{2} .
$$

Substituting (31) into (D.3), we obtain

$$
F S P \leq\left[\left(\xi_{r, r} \lambda_{\max }\left\{\tilde{B}_{r}^{H} \tilde{B}_{r}\right\}\right)^{1 / 2}+\left(\xi_{c, c} \lambda_{\max }\left\{\tilde{B}_{c}^{H} \tilde{B}_{c}\right\}\right)^{1 / 2}\right]^{2}
$$

where $\lambda_{\max }\{Q\}$ denotes the maximal eigenvalue of $Q$. Furthermore, based on (A.11) and the property of Kronecker product [7]

$$
\begin{aligned}
\langle\mathrm{KP} .4\rangle \operatorname{det}\left\{Q_{1}-q_{1} I\right\} & =0, \quad \operatorname{det}\left\{Q_{2}-q_{2} I\right\}=0, \\
\Rightarrow \operatorname{det}\left\{Q_{1} \otimes Q_{2}-q_{1} q_{2} I\right\} & =0
\end{aligned}
$$

where $\operatorname{det}\{Q\}$ denotes the determinant of the matrix $Q$, it can be shown that

$$
\left\{\begin{array}{l}
\lambda_{\max }\left\{\tilde{B}_{c}^{H} \tilde{B}_{c}\right\}=\lambda_{\max }\left\{B_{c}^{H} B_{c}\right\}<\sigma_{c}^{2} \\
\lambda_{\max }\left\{\tilde{B}_{r}^{H} \tilde{B}_{r}\right\}=\lambda_{\max }\left\{B_{r}^{H} B_{r}\right\}<\sigma_{r}^{2} .
\end{array} .\right.
$$

Therefore, we have

$$
F S P<\left(\xi_{c, c}^{1 / 2} \sigma_{c}+\xi_{r, r}^{1 / 2} \sigma_{r}\right)^{2} .
$$

If the interferers are uncorrelated, (33) reveals that both $\xi_{c, c}$ and $\xi_{r, r}$ are not greater than $\sum_{i=2}^{P}\left(\left|d_{c, i}\right|^{2}+\left|d_{r, i}\right|^{2}\right)^{-1}$. Thus, the inequality in (D.6) becomes

$$
F S P<\sum_{i=2}^{P}\left(\left|d_{c, i}\right|^{2}+\left|d_{r, i}\right|^{2}\right)^{-1}\left(\sigma_{c}+\sigma_{r}\right)^{2} .
$$


From (6), it can be shown that $\left|d_{c, i}\right|^{2}>1$ if $\left|u_{i}-u_{1}\right|>1 / 3$. Similarly, we have $\left|d_{r, i}\right|^{2}>1$ if $\left|v_{i}-v_{1}\right|>1 / 3$. Thus, if $\left|u_{i}-u_{1}\right|>1 / 3$ or $\left|v_{i}-v_{1}\right|>1 / 3$, we have $\left|d_{c, i}\right|^{2}+\left|d_{r, i}\right|^{2}>1$ for $i=2,3, \ldots, P$. Hence, (D.7) reduces to

$$
F S P<(P-1)\left(\sigma_{c}+\sigma_{r}\right)^{2} .
$$

Finally, substituting (D.8) into (29) yields the result shown by (34).

\section{REFERENCES}

[1] M. G. Amin, "Concurrent nulling and locations of multiple interference in adaptive antenna arrays," IEEE Trans. Signal Processing, vol. 40, pp. 2658-2663, Nov. 1992.

[2] H. Subbaram and K. Abend, "Interference suppression via orthogonal projections: A performance analysis," IEEE Trans. Antennas Propagat., vol. 41, pp. 1187-1193, Sept. 1993.

[3] B. Friedlander, "A signal subspace method for adaptive interference cancellation," IEEE Trans. Acoust., Speech, Signal Processing, vol. 36, pp. 1835-1845, Dec. 1988.

[4] A. M. Haimovich and Y. Bar-Ness, "An eigenanalysis interference canceler,” IEEE Trans. Signal Processing, vol. 39, pp. 76-84, Jan. 1991.

[5] Y. Bressler, V. U. Reddy, and T. Kailath, "Optimum beamforming for coherent signals and interference," IEEE Trans. Acoust., Speech, Signal Processing, vol. 36, pp. 833-842, June 1988.

[6] K. Ohnishi and R. T. Milton, "A new optimization technique for adaptive antenna arrays," IEEE Trans. Antennas Propagat., vol. 41, pp. 525-532, May 1993.

[7] A. Graham, Kronecker Products and Matrix Calculus with Applications. New York: Ellis Horwood, 1981.

[8] J. E. Hudson, Adaptive Array Principles. New York: Peter Peregrinus, 1981.

[9] F. Li and R. J. Vaccaro, "Unified analysis for DOA estimation algorithms in array signal processing," Signal Processing,vol. 25, pp. 147-169, Nov. 1991.

[10] M. Kaveh and A. J. Barabell, "The statistical performance of the MUSIC and minimum-norm algorithms in resolving plane waves in noise," IEEE Trans. Acoust., Speech, Signal Processing, vol. ASSP-34, pp. 331-341, Apr. 1986.

[11] M. Wax and T. Kailath, "Detection of signals by information theoretic criteria," IEEE Trans. Acoust., Speech, Signal Processing, vol. ASSP-33, pp. 387-392, Apr. 1985.
[12] P. Stoica, "On estimating the noise power in array processing," Signal Processing, vol. 26, pp. 205-220, Feb. 1992.

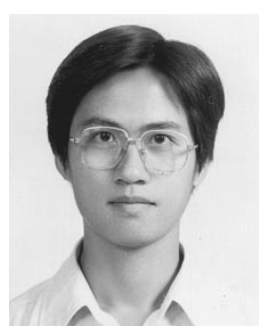

Cheng-Chou Lee was born in Taipei, Taiwan, on July 20, 1969. He received the B.S. and Ph.D. degrees in electrical engineering from the National Taiwan University, Taipei, Taiwan, in 1991 and 1997, respectively.

His current research interests include the adaptive signal processing, array signal processing, and the signal processing in wireless communication systems.

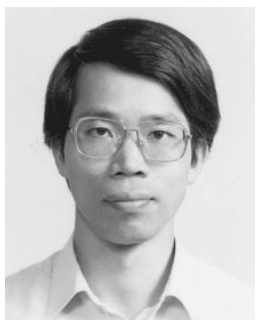

Ju-Hong Lee (S'81-M'83) was born in I-Lan, Taiwan, on December 7, 1952. He received the B.S. degree from the National Cheng-Kung University, Tainan, Taiwan, in 1975, the M.S. degree from the National Taiwan University, Taipei, Taiwan, in 1977, and the Ph.D. Degree from Rensselaer Polytechnic Institute, Troy, NY, in 1984, all in electrical engineering.

From September 1980 to July 1984, he was a Research Assistant and was involved in research on multidimensional recursive digital filtering in the Department of Electrical, Computer, and Systems Engineering at Rensselaer Polytechnic Institute. From August 1984 to July 1986, he was a Visiting Associate Professor and later in August 1986 became an Associate Professor in the Department of Electrical Engineering, National Taiwan University. Since August 1989, he has been a Professor at the same university. He was appointed Visiting Professor in the Department of Computer Science and Electrical Engineering, University of Maryland, Baltimore, during a sabbatical leave in 1996. His current research interests include multidimensional digital signal processing, image processing, detection and estimation theory, analysis and processing of joint vibration signals for the diagnosis of cartilage pathology, and adaptive signal processing and its applications in communications.

Dr. Lee received Outstanding Research Awards from the National Science Council in the academic years of 1988, 1989, and 1991-1994. 